\title{
Reliability, Packaging, Testing, and Characterization of MEMS and MOEMS II
}

This is the second special section on the reliability, packaging, testing, and characterization of MEMS and MOEMS published in the Journal of Micro/Nanolithography, MEMS and MOEMS $\left(\mathrm{JM}^{3}\right)$. Research on the topics of reliability, packaging, testing, and characterization of MEMS/MOEMS is of significant importance to the successful commercialization of these advanced and useful emerging technologies. The main objective of this special section is to provide a publication source for in-depth investigations and interdisciplinary discussions involving reliability, packaging, testing, and characterization of MEMS/MOEMS. The response to the call for papers for this special section was excellent and the resulting group of papers should be technically rewarding for the MEMS/MOEMS community.

This special section covers ohmic contacts to MEMS switches, nanomaterials for sensors, dielectric breakdown characteristics, qualification of FPA-based radiometric packages, deformable mirrors for adaptive optics, microfluidics packaging, dry etching, and pressure sensing.

Some of the papers in this special section were originally presented at the Reliability, Packaging, Testing, and Characterization of MEMS/MOEMS and Nanodevices conference sponsored by SPIE and held in San Jose, California, in 2010.
The papers that were presented at this conference have been elaborated with new substantiating results for this special section.

I would personally like to thank all the reviewers of this special section for their timely support in reviewing the articles for $\mathrm{JM}^{3}$. I also want to thank all the authors and coauthors of this section for their interest in publishing their valuable research efforts in this special section. I want to thank Dr. Burn J. Lin, Editor-in-Chief of $\mathrm{JM}^{3}$, Dr. Thomas Suleski, senior editor, and Dr. Ed Motamedi, senior editor, for providing me with the opportunity to act as the guest editor of this special section. Finally, my deep gratitude is directed to Karolyn Labes, Felicia Andreotta, Rebecca Saxton, Brenda McDonald, and others from the SPIE journals staff for their kind support and assistance in organizing this special section.

\section{Rajeshuni Ramesham}

Jet Propulsion Laboratory

California Institute of Technology

National Aeronautics and Space Administration

\section{Guest Editor}

